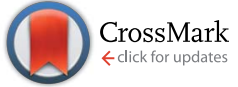

Cite this: RSC Adv., 2016, 6, 78514

DOI: $10.1039 / c 6 r a 90073 a$

www.rsc.org/advances

\section{Correction: Solid phase synthesis and RNA-binding activity of an arginine-containing nucleopeptide}

\author{
G. N. Roviello, ${ }^{\star a}$ C. Vicidomini, ${ }^{a}$ S. Di Gaetano, ${ }^{a}$ D. Capasso, ${ }^{b}$ D. Musumeci ${ }^{\text {ac }}$ \\ and V. Roviello ${ }^{\mathrm{d}}$
}

Correction for 'Solid phase synthesis and RNA-binding activity of an arginine-containing nucleopeptide' by G. N. Roviello et al., RSC Adv., 2016, 6, 14140-14148.

The Acknowledgements section should be revised as shown below:

We thank Professors Antonio Roviello and Daniela Montesarchio (Federico II University, Naples - Italy) for their precious suggestions, and Mr Leopoldo Zona (IBB-CNR, Naples - Italy) for his invaluable technical assistance. We also thank Consiglio Nazionale delle Ricerche (CNR) for the research grant received under the bilateral CNR, Italy-SRNSF, Georgia research Programme (2014-2015), Regione Campania for the research grant received for the research project "Nuovi sistemi nucleopeptidici per applicazioni diagnostiche" under the Legge Regionale no. 5 (annualità 2007) research programme and the European Union's Horizon 2020 research and innovation programme (Marie Sklodowska - Curie Grant Agreement No. 642157).

The Royal Society of Chemistry apologises for these errors and any consequent inconvenience to authors and readers.

${ }^{a}$ CNR, Istituto di Biostrutture e Bioimmagini - (Mezzacannone Site and Headquarters), 80134 Napoli, Italy. E-mail: giovanni.roviello@cnr.it; Fax: +39-081-2534574; Tel: +39081-2534585

${ }^{b}$ Università di Napoli "Federico II", Dipartimento di Farmacia, 80134 Napoli, Italy

"Università di Napoli "Federico II", Dipartimento di Scienze Chimiche, 80126 Napoli, Italy

${ }^{d}$ Università di Napoli "Federico II", Dipartimento di Ingegneria Chimica, dei Materiali e della Produzione Industriale (DICMaPI), 80125 Napoli, Italy 